\title{
Single-cell transcriptional diversity is a hallmark of developmental potential
}

\section{Authors}

Gunsagar S. Gulati ${ }^{1, \dagger}$, Shaheen S. Sikandar ${ }^{1, \dagger}$, Daniel J. Wesche ${ }^{1}$, Anoop Manjunath ${ }^{1}$, Anjan Bharadwaj $^{1}$, Mark J. Berger ${ }^{2}$, Francisco llagan ${ }^{1}$, Angera H. Kuo ${ }^{1}$, Robert W. Hsieh ${ }^{1}$, Shang Cai ${ }^{3}$, Maider Zabala ${ }^{1}$, Ferenc A. Scheeren ${ }^{4}$, Neethan A. Lobo ${ }^{1}$, Dalong Qian ${ }^{1}$, Feiqiao B. Yu ${ }^{5}$, Frederick M. Dirbas ${ }^{6}$, Michael F. Clarke ${ }^{1,7}$, Aaron M. Newman ${ }^{1,8, *}$

\section{Affiliations}

1 Institute for Stem Cell Biology and Regenerative Medicine, Stanford University, Stanford, California, USA.

2 Department of Computer Science, Stanford University, Stanford, California, USA.

3 School of Life Sciences, Westlake University, Zhejiang Province, China.

4 Department of Medical Oncology, Leiden University Medical Center (LUMC), Leiden, the Netherlands.

5 Chan Zuckerberg Biohub, San Francisco, California, USA.

6 Department of Surgery, Stanford Cancer Institute, Stanford University, Stanford, California, USA.

7 Department of Medicine, Stanford University, Stanford, California, USA.

8 Department of Biomedical Data Science, Stanford University, Stanford, California, USA.

†These authors contributed equally.

${ }^{*}$ Corresponding author. Email: amnewman@stanford.edu 


\begin{abstract}
Single-cell RNA-sequencing (scRNA-seq) is a powerful approach for reconstructing cellular differentiation trajectories. However, inferring both the state and direction of differentiation without prior knowledge has remained challenging. Here we describe a simple yet robust determinant of developmental potential — the number of detectably expressed genes per celland leverage this measure of transcriptional diversity to develop a new framework for predicting ordered differentiation states from scRNA-seq data. When evaluated on $\sim 150,000$ single-cell transcriptomes spanning 53 lineages and five species, our approach, called CytoTRACE, outperformed previous methods and $\sim 19,000$ molecular signatures for resolving experimentallyconfirmed developmental trajectories. In addition, it enabled unbiased identification of tissueresident stem cells, including cells with long-term regenerative potential. When used to analyze human breast tumors, we discovered candidate genes associated with less-differentiated luminal progenitor cells and validated GULP1 as a novel gene involved in tumorigenesis. Our study establishes a key RNA-based correlate of developmental potential and provides a new platform for robust delineation of cellular hierarchies (https://cytotrace.stanford.edu).
\end{abstract}




\section{Main Text}

In multicellular organisms, tissues are hierarchically organized into distinct cell types and cellular states with intrinsic differences in function and developmental potential ${ }^{1}$. Common methods for studying cellular differentiation hierarchies, such as lineage tracing and functional transplantation assays, have revealed detailed roadmaps of cellular ontogeny at scales ranging from tissues and organs to entire model organisms $\frac{2-4}{4}$. However, despite the power of these technologies, they cannot be applied to human tissues in vivo and generally require prior knowledge of cell type-specific genetic markers ${ }^{2}$. These limitations have made it difficult to study the developmental organization and cell fate decisions of primary human tissues under normal physiological conditions and during disease.

Single-cell RNA-sequencing (scRNA-seq) has recently emerged as a promising approach to study tissue architecture ${ }^{5,6}$ and cellular differentiation trajectories at high resolution in primary tissue specimens ${ }^{\underline{7}}$. Although a large number of computational methods for predicting lineage trajectories have been described, they generally rely upon (1) a priori knowledge of the starting point (and thus, direction) of the inferred biological process $\frac{8-14}{2}$ and (2) the presence of intermediate cell states to reconstruct the trajectory ${ }^{15,16}$. These requirements, although reasonable in well-established systems and in time-series experiments, can be challenging to satisfy in tissues with poorly understood developmental biology, such as human neoplasms ${ }^{17}$. Moreover, it remains difficult to distinguish quiescent (non-cycling) adult stem cells with longterm regenerative potential from more specialized cells using existing in silico approaches. While gene expression-based models can potentially overcome these limitations (e.g., transcriptional entropy ${ }^{18-20}$, pluripotency-associated gene sets ${ }^{21}$ and machine learning strategies $\underline{\underline{22}}$ ), their relative utility across diverse developmental systems and single-cell sequencing technologies is still unclear.

Here, we profiled nearly 19,000 features of single-cell gene expression data to discover factors that accurately predict cellular differentiation states independently of tissue type, species, and platform. Among the top-performing features, we identified a simple yet surprisingly effective determinant of developmental potential-the number of detectably expressed genes per cell. By leveraging this measure of transcriptional diversity, which was noisy at the single-cell level, we developed a new unsupervised framework for determining ordered differentiation states from single-cell transcriptomes, called CytoTRACE (Cellular (yto) Irajectory Reconstruction

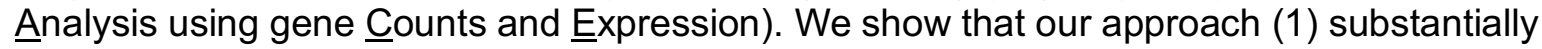
outperforms leading computational methods and 18,706 molecular signatures for predicting differentiation states in 53 experimentally-confirmed developmental trajectories, (2) reveals cellular hierarchies in whole tissues and whole organisms, and (3) identifies key genes associated with stemness and differentiation in both healthy tissues and human cancer. Our results suggest that CytoTRACE can complement existing lineage trajectory tools and aid the identification of immature cells in diverse multicellular systems.

\section{Results}

\section{RNA-based correlates of single-cell differentiation states}

We sought to identify robust, RNA-based determinants of developmental potential without the need for a priori knowledge of developmental direction or intermediate cell states marking cell fate transitions. Toward this end, we evaluated $\sim 19,000$ potential correlates of cell potency in scRNA-seq data, including all available gene sets in the Molecular Signatures Database $(n=$ 
$17,810)^{23}, 896$ gene sets covering transcription factor binding sites from ENCODE ${ }^{24}$ and $\mathrm{ChEA}^{25}$, an mRNA-expression-derived stemness index (mRNAsi) ${ }^{22}$, and three computational techniques that infer stemness as a measure of transcriptional entropy (StemID, SCENT, SLICE $\frac{18-20}{}$ ). We also explored the utility of 'gene counts', or the number of detectably expressed genes per cell, which has been anecdotally observed to correlate with differentiation status ${ }^{26-28}$, but not yet comprehensively evaluated (Methods).

To assess these features, we compiled a training cohort consisting of nine gold standard scRNA-seq datasets with experimentally-confirmed differentiation trajectories. These datasets were selected to prioritize commonly used benchmarking datasets from prior studies $\underline{12,18-20,26, \underline{29}}$ and to ensure a broad sampling of unique developmental states from the mammalian zygote to terminally differentiated cell $s \underline{26,30}$. Overall, the training cohort encompassed 3,174 single cells spanning 49 phenotypes, six tissue types, and three scRNA-seq platforms (Fig. 1A; Methods). To determine performance, the mean value of each feature for all previously annotated cellular phenotypes was calculated and correlated against ground-truth differentiation states. The resulting coefficients (Spearman) were then averaged across the nine training datasets to yield a final score and rank for every feature (Fig. 1B; Methods).

This systematic screen revealed many known and unexpected correlates of differentiation status (Fig. 1C; Fig. S1A). However, one feature in particular showed surprisingly strong performance - the number of detectably expressed genes per cell ('gene counts'). Appearing in the top $1 \%$ of the ranked list (104 out of 18,711$)$, this data-driven feature compared favorably to well-established stemness programs, including cell cycle and pluripotency signatures $\frac{21,22}{\text {, yet }}$ also showed evidence of unique biology and broader applicability. For example, regardless of whether we examined cycling cells, non-cycling cells, or the earliest stages of human embryogenesis prior to the upregulation of pluripotency factors, gene counts generally decreased with successive stages of differentiation (Fig. 1D, left; Fig. S2). Pluripotency genes, by contrast, showed an arc-like pattern during human development, characterized by progressively increasing expression until the emergence of embryonic stem cells, followed by decreasing expression (Fig. 1D, right).

These findings suggested that gene counts might extend beyond isolated experimental systems to recapitulate the full spectrum of cellular ontogeny. To formally test this possibility, we compiled, remapped, and normalized a set of mouse lineage trajectories profiled in vivo by five plate-seq experiments encompassing 5,059 cells and 30 phenotypes that together span all major potency levels $\frac{31}{}$ (Methods). Indeed, when averaged by known phenotypes and assessed across independent studies, the relationship between gene counts and differentiation was robustly maintained $\left(R^{2}=0.89, P<0.0001\right.$; Fig. 1E; Methods).

Given these striking results, we performed a series of experiments to better understand the biological basis of gene counts and the factors that influence its measurement.

\section{Robustness and biological basis of gene counts}

We started by characterizing the robustness of gene counts to variation in two key technical parameters: (1) sparsity in single-cell gene expression data and (2) the number of sequenced reads per cell. To investigate the former, we compared gene counts derived from single-cell transcriptomes with gene counts derived from bulk RNA-seq profiles ${ }^{32}$, pooled single-cell transcriptomes, and single-cell transcriptomes following missing value imputation $\underline{33}$. Regardless of the approach, we observed significantly reduced performance for predicting differentiation states when attempting to overcome sparsity (Fig. S3A-D). This suggests that sparsity in 
scRNA-seq data is driven by real biological heterogeneity in addition to technical noise. Such heterogeneity, while informative for gene counts as a measure of developmental potential, is lost or severely degraded at the population level (Fig. S3B, E).

We next examined the relationship between gene counts and the number of reads per cell. Reanalysis of seven scRNA-seq experiments profiled by plate-based protocols showed that even when down-sampling to 10,000 reads per cell, the predictive performance of gene counts was largely maintained (Fig. S4A-C). Moreover, the mean number of detectably expressed genes per dataset was linearly related to the logarithm of the mean number of reads (mean $R^{2}=$ 0.99; Fig. S4D). As a result, for most datasets, variation in gene counts due to fluctuations in the number of reads was minimal. Furthermore, predictive performance was only modestly impacted when varying the expression threshold for calculating the number of expressed genes per cell and was unaffected by the removal of potential doublets (Fig. S5A, B).

To investigate potential biological correlates of gene counts, we next compared it with the number of detectable mRNA molecules per cell, as measured by unique molecular identifiers (UMIs), external spike-in standards (ERCCs), and Census, a statistical approach to infer the number of mRNA transcripts that are available for capture following cell lysis $\stackrel{34}{ }$. By analyzing UMI ( $n=14$ datasets) and ERCC ( $n=7$ datasets) data from previously published droplet-based and plate-based experiments, respectively, we found that a large proportion of the variance in gene counts could be attributed to single-cell mRNA content alone (UMI: mean $R^{2}=0.84$; ERCC: mean $R^{2}=0.29$; Fig. S6A, B). This relationship was further confirmed in 17 non-UMI

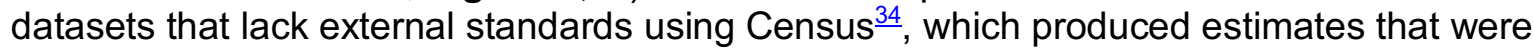
nearly perfectly correlated with the number of unique detectable genes (i.e., canonical transcripts) per cell (mean $R^{2} \approx 1$ ) (Fig. S6C; Methods). We also measured the linear association between gene counts and the number of unique protein-coding splice isoforms per cell. As expected, across 10 plate-seq datasets, gene counts and mRNA diversity were tightly interrelated (mean $R^{2}=0.98$; Fig. 2A; Fig. S6D).

Since the transcriptional output of a cell is associated with its genome-wide chromatin profile, we hypothesized that single-cell gene counts might ultimately be a surrogate for global chromatin accessibility, which has been previously shown to decrease with differentiation ${ }^{35-38}$. To test this, we compared single-cell gene counts derived from scRNA-seq data with paired bulk ATAC-seq (assay for transposase-accessible chromatin sequencing) profiles obtained from a recent study of in vitro mesodermal differentiation from human embryonic stem cells (hESCs) ${ }^{32}$ (Fig. 2B; Fig. S7A). In support of our hypothesis, genome-wide chromatin accessibility was observed to progressively decrease with differentiation of hESCs into paraxial mesoderm and lateral mesoderm lineages (Fig. 2B; Fig. S7A). Moreover, when segregated by developmental lineage, we observed strong concordance between the number of accessible peaks and the mean number of detectably expressed genes per phenotype (Fig. 2C; Fig. S7B, C).

\section{Development of CytoTRACE}

Although gene counts generally showed robust performance when averaged by known phenotypes, in some datasets, such as the in vitro differentiation of hESCs into the gastrulation layers $^{39}$, it exhibited considerable intra-phenotypic variation (Fig. 3A, left). In fact, when evaluated at a single-cell level, 412 predefined gene sets from our in silico screen outperformed gene counts (Fig. S1B). Since scRNA-seq was designed to capture single-cell gene expression, we reasoned that genes whose expression patterns correlate with gene counts might better capture differentiation states. Remarkably, by simply taking the geometric mean of genes that were most correlated with gene counts in each dataset (Fig. S8A-C; Methods), the resulting 
dataset-specific 'gene counts signature' (GCS) became the top-performing measure in the screen, outranking every pre-defined molecular profile and computational tool that we assessed (Fig. S1B).

Although GCS is likely to be influenced by multi-lineage priming in some settings $\underline{40}$, it is derived from all detectably expressed genes per cell in a given dataset. It is therefore inherently robust to drop-out events, agnostic to prior knowledge of developmentally regulated genes, and not solely attributable to a previously defined molecular signature (e.g., pluripotency; Fig. 1D). Nevertheless, GCS was still moderately noisy in some datasets (e.g., Fig. 3A, center; Fig. S8A-C). We therefore implemented a novel two-step procedure to directly smooth GCS on the basis of transcriptional covariance among single cells (Fig. 3A, right; Fig. S8A-D; Methods). The resulting method, which we call CytoTRACE (Cellular (yto) Trajectory Reconstruction

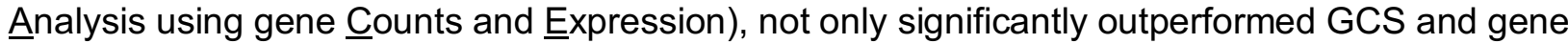
counts (Fig. S8A), but also outperformed all evaluated features by a considerable margin (Fig. S1B).

\section{Performance evaluation across tissues, species, and platforms}

To validate our findings, we assembled a greatly expanded compendium of 33 additional scRNA-seq datasets. These data were selected to represent diverse experimentally-confirmed developmental lineages and consisted of 141,267 single cells spanning 266 phenotypes, 11 tissue types, five species, nine scRNA-seq platforms (three droplet-based and six plate-based protocols, ranging from an average of $\sim 10,000$ to $\sim 1 \mathrm{M}$ UMls or reads per cell, respectively; Fig. S4A), and 26 studies (Fig. 3B; Methods). As before, performance was determined using Spearman correlations to determine concordance against ground truth data at the single-cell level and by phenotype (Methods).

When assessed at the single-cell level, CytoTRACE markedly outperformed existing methods and gene sets (Fig. 3C, D; Fig. S9A), and was positively correlated with the direction of differentiation in $88 \%$ of datasets $\left(P=7 \times 10^{-7}\right.$, Binomial test). These results were consistent with our findings in the training cohort (Fig. 3C; Fig. S9B). Moreover, comparable results were obtained on datasets with discontinuous developmental processes lacking transitional cells (Fig. S10A, C). These data distinguish CytoTRACE from RNA velocity, a recently described kinetic model that can predict future cell states, but is limited to scRNA-seq data with continuous fate transitions and genes with mRNA half-lives on the order of hours $\frac{15}{1}$ (Fig. S10B, D). Importantly, no significant biases in CytoTRACE performance were observed in relation to tissue type, species, the number of cells analyzed, time-series experiments versus snapshots of developmental states, or plate-based versus droplet-based technologies (Fig. S11).

\section{Differentiation-associated genes and cellular hierarchies}

Given CytoTRACE's ability to faithfully recover the direction of differentiation in nearly every evaluated dataset, we asked whether it might prove useful for discovering genetic markers of immature cells without prior knowledge of cellular phenotypes. Toward this end, we rankordered all genes in each benchmarking dataset based on their correlation with CytoTRACE and defined 'ground truth' gene sets that marked the least and most differentiated cells as annotated in the original studies. In $86 \%$ of datasets, these gene sets were significantly skewed in the correct direction toward the extreme ends of the ranked transcriptome (adjusted $P<0.05$, GSEA ${ }^{41}$; Fig. 3E; Fig. S12). Moreover, CytoTRACE automatically prioritized well-established stem and progenitor markers, including Kit and Stmn1 in the mouse bone marrow ${ }^{42}$ and Axin2 
and $\operatorname{Lgr} 5$ in mouse intestinal crypts ${ }^{43}$, underscoring the utility of CytoTRACE for the de novo discovery of developmentally-regulated genes (Fig. 3E).

We next explored the potential of CytoTRACE to complement existing techniques for trajectory visualization and branch detection. By combining it with a two-dimensional force-directed layout algorithm to analyze 39,505 cells from zebrafish embryos, CytoTRACE readily revealed complex branching patterns arising during whole organism development from a fertilized egg (Fig. S13) $)^{44}$. Likewise, when applied to 3,427 unselected mouse bone marrow cells ${ }^{42}$, CytoTRACE enabled reconstruction of the directionality and lineage structure of hematopoietic development (Fig. 4A).

Complex lineage relationships in scRNA-seq data can also be determined by dedicated branch detection tools $\frac{14}{4}$, such as Monocle $2^{\frac{34}{4}}$, however these approaches do not predict the starting point of the biological process. For example, when applied to 4,442 bone marrow cells $\frac{42}{\text {, }}$ Monocle 2 identified 23 possible "roots" from which to calculate pseudotime values (Fig. 4B, left). Only 1 of these 23 states is correct (4\% of possibilities), which we define as the state that is most enriched for previously annotated stem and progenitor cells (state 12 in Fig. 4B). By integrating CytoTRACE with Monocle 2, the correct root was readily identified without user input (Fig. 4B, right; Fig. S14A, B). This facilitated identification of lineage-specific regulatory factors and marker genes during granulocyte, monocyte, and B cell differentiation (Fig. S14C). Similarly, when CytoTRACE and Monocle 2 were applied to 4,581 mouse intestinal cells $\underline{43}$, we were able to automatically determine the root, developmental ordering, and branching processes of stem and progenitor cells differentiating into enterocyte and secretory lineages (Fig. S14D-E).

\section{Dissection of stem cell and progenitor populations}

We next asked whether CytoTRACE could distinguish cycling and long-term/quiescent stem cells from their downstream progenitors ${ }^{45,46}$. As these populations are well-characterized in the bone marrow ${ }^{3}$, we investigated this question in the mouse hematopoietic system. While both cycling and quiescent hematopoietic stem cell (HSC) subpopulations $\underline{45}$, $\underline{ }$. were correctly predicted to be less differentiated, only proliferative HSCs were significantly ranked above early progenitors (Fig. 4C). This result was not unexpected, however, since quiescent cells have reduced metabolic activity and low RNA content ${ }^{1}$. By devising a simple approach to visualize inferred RNA content as a function of CytoTRACE, we observed a distinct valley in RNA abundance that coincided with elevated expression of Hoxb5, a recently described marker of long-term/quiescent HSCs ${ }^{47}$ (Fig. 4D, E). This analysis further confirms the value of CytoTRACE and suggests a novel approach for elucidating tissue-specific stem cells from scRNA-seq data.

\section{Application to neoplastic disease}

Having validated CytoTRACE's technical performance, we next applied it to a system where developmental trajectories are less well-characterized. A growing body of evidence suggests that human breast tumors are hierarchically organized and originate from subpopulations of cancer cells, called tumor-initiating cells, which are less differentiated, resistant to therapy, and implicated in relapse and metastasis ${ }^{48,49}$. In breast cancer, subpopulations of tumor cells within the luminal progenitor (LP) epithelium are thought to give rise to aggressive basal-like breast cancers, such as triple-negative breast cancer (TNBC) $)^{50,51}$, and possibly also to estrogen

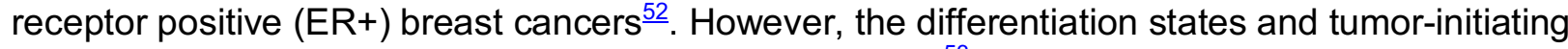
properties of LP subsets remain incompletely understood $\underline{53}$. 
To test whether CytoTRACE can facilitate new insights into immature LP cells and their associated genes in breast cancer, we performed scRNA-seq profiling of breast tumor epithelial cells and adjacent normal epithelial cells from 8 patients with basal-like $(n=2)$ or luminal-like $(n$ = 6) breast cancer. Using a Smart-seq2 protocol combined with fluorescence-activated cell sorting (FACS), we index-sorted and sequenced cells from three major human epithelial subpopulations: basal (CD49f $f^{\text {high }}$ EPCAM $\left.{ }^{\text {med-low }}\right)$, luminal progenitor $\left(C D 49 f^{\text {high }}\right.$ EPCAM $\left.^{\text {high }}\right)$, and mature luminal subpopulations (ML) $\left(C D 49 f^{\text {low }}\right.$ EPCAM $\left.^{\text {high }}\right)$ (Fig. S15A). After removing low quality cells and applying principal component analysis to visualize the data, we confirmed three well-separated clusters of basal, LP, and ML cells, each with characteristic expression patterns of previously described lineage markers (Fig. 5A; Fig. S15B). No obvious clustering was observed for tumor/normal differences or by patient (Fig. 5A).

To validate the ability of CytoTRACE to define LP differentiation states, we started by rankordering genes in adjacent normal LPs by their correlation with CytoTRACE (Fig. 5B, top). We found that previously described marker genes of less differentiated normal LPS (ALDH1A3 and MFGE8) ${ }^{54,55}$ and more differentiated normal LPs (GATA3, FOXA1, and $A R^{54,56}$ ) were successfully enriched by this approach (Fig. 5B, bottom). Moreover, genes that were upregulated in highly clonogenic normal $\mathrm{LPs}^{\frac{54}{4}}$ were skewed toward genes predicted to mark less differentiated cells (Fig. 5B, bottom).

Given these favorable results, we next sought to identify LP genes associated with tumorigenesis. By rank-ordering LP genes in malignant cells by their correlation with CytoTRACE (Fig. 5C, top), we observed a highly significant enrichment of genes whose knockdown by RNA interference (RNAi) led to decreased viability of tumor cells in patientderived xenograft (PDX) models of $\operatorname{TNBC}^{\underline{57}}(Q=0.002$, GSEA; Fig. 5C, bottom; Fig. S16). Moreover, when we applied CytoTRACE to prioritize genes in tumor LPs as compared to tumor MLs, the latter of which are developmentally downstream of LPs in normal breast $\frac{54,58}{}$, the top 15 genes included known members of tumorigenic pathways in breast cancer (e.g., MET, JAK1, and $X B P 1^{59-61}$ ), as well as novel candidates (e.g., GULP1) (Fig. 5D, top). To further refine this list, we focused on genes that were (1) more highly expressed in tumor LPs than MLs and (2) expressed in a subpopulation of tumor LPs ( $<20 \%$ of cells) (Fig. 5D, bottom). After applying this filter, GULP1 emerged as the top candidate (Fig. 5D, bottom right).

GULP1 is an engulfment adaptor protein and is the human homolog of Drosophila Ced6 $\underline{62}$. Moreover, the murine homolog of GULP1 is a specific marker of mouse HSCs, suggesting a possibly conserved role of this gene in other immature cell states (Fig. S17A). Since the role of GULP1 in human breast cancer has not been established, we measured the effect of GULP1 knockdown on the proliferation of metastatic TNBC cell lines, MDA-MB-231 and MDA-MB-468, compared to an empty vector control (Fig. S17B-E). GULP1 knockdown significantly reduced proliferation of MDA-MB-231 and MDA-MB-468 as measured by a colorimetric assay for metabolic activity (Fig. S17E). Next, we tested the effect of GULP1 knockdown in PDXs from $\mathrm{ER}+$ and TNBC patients from our single-cell cohort (Fig. 5E). We found that knockdown of GULP1 significantly reduced tumor growth in the TNBC sample and completely abrogated the ER+ tumor compared to empty vector control (Fig. 5F).

Taken together, these data suggest a novel role for GULP1 in human breast cancer tumorigenesis and demonstrate the promise of CytoTRACE for the discovery of malignant cell differentiation states and new therapeutic targets. 


\section{Discussion}

Efforts to characterize single-cell transcriptomes in diverse tissues, organs, and whole organisms ${ }^{42,63}$ have underscored the need for robust RNA-based determinants of developmental potential. In our analysis of $\sim 19,000$ features across 42 developmental processes and nearly 150,000 single cells, we found that gene counts, or the number of detectably expressed genes per cell, powerfully associates with transcriptional diversity and differentiation status. Although anecdotally observed in specific experimental systems (mouse alveolar epithelial development, zebrafish thrombopoiesis, and neuron differentiation from $\mathrm{hESCs} \frac{26-28}{}$ ), we demonstrate for the first time that this association (1) outperforms most stemness inference tools and pre-defined molecular signatures from a compendium of nearly 19,000 RNA-based features, (2) is generally independent of species, platform, and tissue type, and (3) is broadly applicable throughout cellular ontogenesis.

Although previous studies have demonstrated a global reduction in chromatin accessibility and/or plasticity during lineage commitment in specific developmental settings (e.g. embryonic

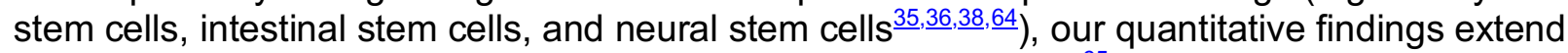
the scope of this result. Moreover, as has been previously shown $\underline{65}$, our data indicate that variability in gene counts between phenotypically identical single cells is not exclusively due to drop-out events, but also due to differential sampling of the transcriptome (Fig. S3). Our data are therefore consistent with a model in which less mature cells maintain looser chromatin to permit wider sampling of the transcriptome, while more differentiated cells generally restrict chromatin accessibility and transcriptional diversity as they specialize (Fig. S6C) ${ }^{66}$. Future studies will be needed to further confirm the validity of this model and assess its relevance across diverse tissue compartments, developmental time points, and phenotypic states.

The identification of gene counts as a leading measure of cellular differentiation status motivated the development of CytoTRACE, a computational framework that leverages and significantly improves upon gene counts for resolving differentiation states at the single-cell level. Unlike most existing methods for lineage trajectory analysis $\frac{8-15}{}$, however, CytoTRACE can predict both the relative state and direction of differentiation in a manner that is independent of specific timescales or the presence of continuous developmental processes in the data. CytoTRACE is also agnostic to tissue type, species, and scRNA-seq platform.

We anticipate that these advantages will enable significant applications. For example, by using CytoTRACE to analyze scRNA-seq profiles of human breast tumors, we identified new candidate genes associated with less-differentiated luminal progenitor cells and established a novel role for GULP1 in breast tumorigenesis. These data emphasize the utility of CytoTRACE for characterizing tumor differentiation hierarchies and for discovering novel biomarkers and therapeutic targets. Furthermore, by integrating RNA content with CytoTRACE, we demonstrated, for the first time to our knowledge, that quiescent adult stem cells can be distinguished from downstream progenitors using an unsupervised in silico approach. Given the immense regenerative potential of quiescent stem cells, their identification in human tissues has broad implications in regenerative medicine and cancer treatment.

While CytoTRACE can recapitulate developmental orderings from single lineages to whole organisms, several challenges remain. For example, although the direction of differentiation was predicted correctly in nearly all datasets, $12 \%$ of cases were mischaracterized. These datasets also proved problematic for other approaches, suggesting there may be opportunities for future enhancements. In addition, CytoTRACE is currently expressed in rank space, which is not 
directly comparable across different datasets. Efforts to overcome these challenges are underway.

In summary, we conclude that the number of expressed genes per cell is a hallmark of developmental potential. By exploiting this data-driven property of scRNA-seq data, we developed a broadly applicable framework for resolving single-cell differentiation hierarchies. We envision that our approach will complement existing scRNA-seq analysis strategies, with implications for the identification of immature cells and their developmental trajectories in complex tissues throughout multicellular life. 


\section{References}

1 Visvader, J. E. \& Clevers, H. Tissue-specific designs of stem cell hierarchies. Nat Cell Biol 18, 349-355, doi:10.1038/ncb3332 (2016).

$2 \quad$ Kretzschmar, K. \& Watt, F. M. Lineage tracing. Cell 148, 33-45, doi:10.1016/j.cell.2012.01.002 (2012).

3 Seita, J. \& Weissman, I. L. Hematopoietic stem cell: self-renewal versus differentiation. Wiley Interdiscip Rev Syst Biol Med 2, 640-653, doi:10.1002/wsbm.86 (2010).

4 Sulston, J. E., Schierenberg, E., White, J. G. \& Thomson, J. N. The embryonic cell lineage of the nematode Caenorhabditis elegans. Dev Biol 100, 64-119 (1983).

5 Cao, J. et al. Comprehensive single-cell transcriptional profiling of a multicellular organism. Science 357, 661-667, doi:10.1126/science.aam8940 (2017).

6 Jaitin, D. A. et al. Massively parallel single-cell RNA-seq for marker-free decomposition of tissues into cell types. Science 343, 776-779, doi:10.1126/science.1247651 (2014).

$7 \quad$ Kester, L. \& van Oudenaarden, A. Single-Cell Transcriptomics Meets Lineage Tracing. Cell Stem Cell 23, 166-179, doi:10.1016/j.stem.2018.04.014 (2018).

8 Campbell, K., Ponting, C. P. \& Webber, C. Laplacian eigenmaps and principal curves for high resolution pseudotemporal ordering of single-cell RNA-seq profiles. bioRxiv (2015).

9 Qiu, X. et al. Reversed graph embedding resolves complex single-cell trajectories. Nat Methods 14, 979-982, doi:10.1038/nmeth.4402 (2017).

10 Cannoodt, R. et al. SCORPIUS improves trajectory inference and identifies novel modules in dendritic cell development. bioRxiv (2016).

11 Street, K. et al. Slingshot: cell lineage and pseudotime inference for single-cell transcriptomics. BMC Genomics 19, 477, doi:10.1186/s12864-018-4772-0 (2018).

12 Chen, $\mathrm{H}$. et al. STREAM: Single-cell Trajectories Reconstruction, Exploration And Mapping of omics data. bioRxiv (2018).

$13 \mathrm{Ji}, \mathrm{Z}$. \& Ji, H. TSCAN: Pseudo-time reconstruction and evaluation in single-cell RNA-seq analysis. Nucleic Acids Res 44, e117, doi:10.1093/nar/gkw430 (2016).

14 Saelens, W., Cannoodt, R., Todorov, H. \& Saeys, Y. A comparison of single-cell trajectory inference methods: towards more accurate and robust tools. bioRxiv (2018).

15 La Manno, G. et al. RNA velocity of single cells. Nature 560, 494-498, doi:10.1038/s41586-018-0414-6 (2018).

16 Shin, J. et al. Single-Cell RNA-Seq with Waterfall Reveals Molecular Cascades underlying Adult Neurogenesis. Cell Stem Cell 17, 360-372, doi:10.1016/j.stem.2015.07.013 (2015).

17 Pattabiraman, D. R. \& Weinberg, R. A. Tackling the cancer stem cells - what challenges do they pose? Nat Rev Drug Discov 13, 497-512, doi:10.1038/nrd4253 (2014).

18 Grun, D. et al. De Novo Prediction of Stem Cell Identity using Single-Cell Transcriptome Data. Cell Stem Cell 19, 266-277, doi:10.1016/j.stem.2016.05.010 (2016).

19 Teschendorff, A. E. \& Enver, T. Single-cell entropy for accurate estimation of differentiation potency from a cell's transcriptome. Nat Commun 8, 15599, doi:10.1038/ncomms15599 (2017).

20 Guo, M., Bao, E. L., Wagner, M., Whitsett, J. A. \& Xu, Y. SLICE: determining cell differentiation and lineage based on single cell entropy. Nucleic Acids Res 45, e54, doi:10.1093/nar/gkw1278 (2017).

21 Muller, F. J. et al. Regulatory networks define phenotypic classes of human stem cell lines. Nature 455, 401-405, doi:10.1038/nature07213 (2008).

22 Malta, T. M. et al. Machine Learning Identifies Stemness Features Associated with Oncogenic Dedifferentiation. Cell 173, 338-354 e315, doi:10.1016/j.cell.2018.03.034 (2018). 
23 Liberzon, A. et al. The Molecular Signatures Database (MSigDB) hallmark gene set collection. Cell Syst 1, 417-425, doi:10.1016/j.cels.2015.12.004 (2015).

24 Gerstein, M. B. et al. Architecture of the human regulatory network derived from ENCODE data. Nature 489, 91-100, doi:10.1038/nature11245 (2012).

25 Lachmann, A. et al. ChEA: transcription factor regulation inferred from integrating genome-wide ChIP-X experiments. Bioinformatics 26, 2438-2444, doi:10.1093/bioinformatics/btq466 (2010).

26 Wang, J. et al. Single-cell gene expression analysis reveals regulators of distinct cell subpopulations among developing human neurons. Genome Res 27, 1783-1794, doi:10.1101/gr.223313.117 (2017).

27 Treutlein, B. et al. Reconstructing lineage hierarchies of the distal lung epithelium using single-cell RNA-seq. Nature 509, 371-375, doi:10.1038/nature13173 (2014).

28 Macaulay, I. C. et al. Single-Cell RNA-Sequencing Reveals a Continuous Spectrum of Differentiation in Hematopoietic Cells. Cell Rep 14, 966-977, doi:10.1016/j.celrep.2015.12.082 (2016).

29 Trapnell, C. et al. The dynamics and regulators of cell fate decisions are revealed by pseudotemporal ordering of single cells. Nat Biotechnol 32, 381-386, doi:10.1038/nbt.2859 (2014).

30 Olsson, A. et al. Single-cell analysis of mixed-lineage states leading to a binary cell fate choice. Nature 537, 698-702, doi:10.1038/nature19348 (2016).

31 Jaenisch, R. \& Young, R. Stem cells, the molecular circuitry of pluripotency and nuclear reprogramming. Cell 132, 567-582, doi:10.1016/j.cell.2008.01.015 (2008).

32 Loh, K. M. et al. Mapping the Pairwise Choices Leading from Pluripotency to Human Bone, Heart, and Other Mesoderm Cell Types. Cell 166, 451-467, doi:10.1016/j.cell.2016.06.011 (2016).

33 Hochgerner, H., Zeisel, A., Lonnerberg, P. \& Linnarsson, S. Conserved properties of dentate gyrus neurogenesis across postnatal development revealed by single-cell RNA sequencing. Nat Neurosci 21, 290-299, doi:10.1038/s41593-017-0056-2 (2018).

34 Qiu, X. et al. Single-cell mRNA quantification and differential analysis with Census. Nat Methods 14, 309-315, doi:10.1038/nmeth.4150 (2017).

35 Aughey, G. N., Estacio Gomez, A., Thomson, J., Yin, H. \& Southall, T. D. CATaDa reveals global remodelling of chromatin accessibility during stem cell differentiation in vivo. Elife 7, doi:10.7554/eLife.32341 (2018).

36 Gomez, N. C. et al. Widespread Chromatin Accessibility at Repetitive Elements Links Stem Cells with Human Cancer. Cell Rep 17, 1607-1620, doi:10.1016/j.celrep.2016.10.011 (2016).

37 Kornberg, R. D. \& Lorch, Y. Irresistible force meets immovable object: transcription and the nucleosome. Cell 67, 833-836 (1991).

38 Pekowska, A. et al. Gain of CTCF-Anchored Chromatin Loops Marks the Exit from Naive Pluripotency. Cell Syst 7, 482-495 e410, doi:10.1016/j.cels.2018.09.003 (2018).

39 Chu, L. F. et al. Single-cell RNA-seq reveals novel regulators of human embryonic stem cell differentiation to definitive endoderm. Genome Biol 17, 173, doi:10.1186/s13059016-1033-x (2016).

40 Huang, S., Guo, Y. P., May, G. \& Enver, T. Bifurcation dynamics in lineage-commitment in bipotent progenitor cells. Dev Biol 305, 695-713, doi:10.1016/j.ydbio.2007.02.036 (2007).

41 Sergushichev, A. An algorithm for fast preranked gene set enrichment analysis using cumulative statistic calculation. bioRxiv (2016).

42 Tabula Muris, C. et al. Single-cell transcriptomics of 20 mouse organs creates a Tabula Muris. Nature 562, 367-372, doi:10.1038/s41586-018-0590-4 (2018). 
43 Haber, A. L. et al. A single-cell survey of the small intestinal epithelium. Nature 551, 333339, doi:10.1038/nature24489 (2017).

44 Farrell, J. A. et al. Single-cell reconstruction of developmental trajectories during zebrafish embryogenesis. Science 360, doi:10.1126/science.aar3131 (2018).

45 Srour, E. F. \& Jordan, C. T. Isolation and characterization of primitive hematopoietic cells based on their position in the cell cycle. Methods Mol Med 63, 93-111, doi:10.1385/1-59259-140-X:093 (2002).

46 Wilson, A. et al. Hematopoietic stem cells reversibly switch from dormancy to selfrenewal during homeostasis and repair. Cell 135, 1118-1129, doi:10.1016/j.cell.2008.10.048 (2008).

47 Chen, J. Y. et al. Hoxb5 marks long-term haematopoietic stem cells and reveals a homogenous perivascular niche. Nature 530, 223-227, doi:10.1038/nature16943 (2016).

48 Reya, T., Morrison, S. J., Clarke, M. F. \& Weissman, I. L. Stem cells, cancer, and cancer stem cells. Nature 414, 105-111, doi:10.1038/35102167 (2001).

49 Clarke, M. F. et al. Cancer stem cells--perspectives on current status and future directions: AACR Workshop on cancer stem cells. Cancer Res 66, 9339-9344, doi:10.1158/0008-5472.CAN-06-3126 (2006).

$50 \mathrm{Lim}$, E. et al. Aberrant luminal progenitors as the candidate target population for basal tumor development in BRCA1 mutation carriers. Nat Med 15, 907-913, doi:10.1038/nm.2000 (2009).

51 Sikandar, S. S. et al. Role of epithelial to mesenchymal transition associated genes in mammary gland regeneration and breast tumorigenesis. Nat Commun 8, 1669, doi:10.1038/s41467-017-01666-2 (2017).

52 Polyak, K. Breast cancer: origins and evolution. J Clin Invest 117, 3155-3163, doi:10.1172/JCI33295 (2007).

53 Visvader, J. E. \& Lindeman, G. J. Cancer stem cells in solid tumours: accumulating evidence and unresolved questions. Nat Rev Cancer 8, 755-768, doi:10.1038/nrc2499 (2008).

54 Shehata, M. et al. Phenotypic and functional characterisation of the luminal cell hierarchy of the mammary gland. Breast Cancer Res 14, R134, doi:10.1186/bcr3334 (2012).

55 Ginestier, C. et al. ALDH1 is a marker of normal and malignant human mammary stem cells and a predictor of poor clinical outcome. Cell Stem Cell 1, 555-567, doi:10.1016/j.stem.2007.08.014 (2007).

56 Kouros-Mehr, H., Slorach, E. M., Sternlicht, M. D. \& Werb, Z. GATA-3 maintains the differentiation of the luminal cell fate in the mammary gland. Cell 127, 1041-1055, doi:10.1016/j.cell.2006.09.048 (2006).

57 Hsieh, R. W. et al. CDK19 is a Regulator of Triple-Negative Breast Cancer Growth. bioRxiv (2018).

58 Stingl, J., Eaves, C. J., Zandieh, I. \& Emerman, J. T. Characterization of bipotent mammary epithelial progenitor cells in normal adult human breast tissue. Breast Cancer Res Treat 67, 93-109 (2001).

59 Graveel, C. R., Tolbert, D. \& Vande Woude, G. F. MET: a critical player in tumorigenesis and therapeutic target. Cold Spring Harb Perspect Biol 5,

doi:10.1101/cshperspect.a009209 (2013).

60 Chang, Q. et al. The IL-6/JAK/Stat3 feed-forward loop drives tumorigenesis and metastasis. Neoplasia 15, 848-862 (2013).

61 Chen, X. et al. XBP1 promotes triple-negative breast cancer by controlling the HIF1alpha pathway. Nature 508, 103-107, doi:10.1038/nature13119 (2014).

62 Liu, Q. A. \& Hengartner, M. O. Human CED-6 encodes a functional homologue of the Caenorhabditis elegans engulfment protein CED-6. Curr Bio/ 9, 1347-1350 (1999). 
63 Regev, A. et al. The Human Cell Atlas. bioRxiv (2017).

64 Gorkin, D. U., Leung, D. \& Ren, B. The 3D genome in transcriptional regulation and pluripotency. Cell Stem Cell 14, 762-775, doi:10.1016/j.stem.2014.05.017 (2014).

65 Marinov, G. K. et al. From single-cell to cell-pool transcriptomes: stochasticity in gene expression and RNA splicing. Genome Res 24, 496-510, doi:10.1101/gr.161034.113 (2014).

66 Melcer, S. \& Meshorer, E. Chromatin plasticity in pluripotent cells. Essays In Biochemistry 48, 245-262, doi:10.1042/bse0480245 (2010).

67 Gazit, R. et al. Fgd5 identifies hematopoietic stem cells in the murine bone marrow. J Exp Med 211, 1315-1331, doi:10.1084/jem.20130428 (2014).

68 Yu, G., Wang, L. G., Han, Y. \& He, Q. Y. clusterProfiler: an R package for comparing biological themes among gene clusters. OMICS 16, 284-287, doi:10.1089/omi.2011.0118 (2012).

69 Kowalczyk, M. S. et al. Single-cell RNA-seq reveals changes in cell cycle and differentiation programs upon aging of hematopoietic stem cells. Genome Res 25, 18601872, doi:10.1101/gr.192237.115 (2015).

70 Du, Y., Guo, M., Whitsett, J. A. \& Xu, Y. 'LungGENS': a web-based tool for mapping single-cell gene expression in the developing lung. Thorax 70, 1092-1094, doi:10.1136/thoraxjnl-2015-207035 (2015).

71 Johnson, W. E., Li, C. \& Rabinovic, A. Adjusting batch effects in microarray expression data using empirical Bayes methods. Biostatistics 8, 118-127, doi:10.1093/biostatistics/kxj037 (2007).

72 sva: Surrogate Variable Analysis (R package, 2018).

73 Nakamura, T. et al. A developmental coordinate of pluripotency among mice, monkeys and humans. Nature 537, 57-62, doi:10.1038/nature19096 (2016).

$74 \quad$ Plass, M. et al. Cell type atlas and lineage tree of a whole complex animal by single-cell transcriptomics. Science 360, doi:10.1126/science.aaq1723 (2018).

75 Zheng, G. X. et al. Massively parallel digital transcriptional profiling of single cells. Nat Commun 8, 14049, doi:10.1038/ncomms14049 (2017).

76 Yuzwa, S. A. et al. Developmental Emergence of Adult Neural Stem Cells as Revealed by Single-Cell Transcriptional Profiling. Cell Rep 21, 3970-3986, doi:10.1016/j.celrep.2017.12.017 (2017).

77 Kernfeld, E. M. et al. A Single-Cell Transcriptomic Atlas of Thymus Organogenesis Resolves Cell Types and Developmental Maturation. Immunity 48, 1258-1270 e1256, doi:10.1016/j.immuni.2018.04.015 (2018).

78 Yan, L. et al. Single-cell RNA-Seq profiling of human preimplantation embryos and embryonic stem cells. Nat Struct Mol Biol 20, 1131-1139, doi:10.1038/nsmb.2660 (2013).

79 Liberzon, A. et al. Molecular signatures database (MSigDB) 3.0. Bioinformatics 27, 1739-1740, doi:10.1093/bioinformatics/btr260 (2011).

80 Subramanian, A. et al. Gene set enrichment analysis: a knowledge-based approach for interpreting genome-wide expression profiles. Proc Natl Acad Sci U S A 102, 1554515550, doi:10.1073/pnas.0506580102 (2005).

81 Hanzelmann, S., Castelo, R. \& Guinney, J. GSVA: gene set variation analysis for microarray and RNA-seq data. BMC Bioinformatics 14, 7, doi:10.1186/1471-2105-14-7 (2013).

82 Pasek, J. weights: Weighting and Weighted Statistics. $R$ package version 0.90 (2018).

83 Liu, Y. et al. Transcriptional landscape of the human cell cycle. Proc Natl Acad Sci U S A 114, 3473-3478, doi:10.1073/pnas.1617636114 (2017). 
84 Patro, R., Duggal, G., Love, M. I., Irizarry, R. A. \& Kingsford, C. Salmon provides fast and bias-aware quantification of transcript expression. Nat Methods 14, 417-419, doi:10.1038/nmeth.4197 (2017).

85 Ziegenhain, C. et al. Comparative Analysis of Single-Cell RNA Sequencing Methods. Mol Cell 65, 631-643 e634, doi:10.1016/j.molcel.2017.01.023 (2017).

86 Deng, Q., Ramskold, D., Reinius, B. \& Sandberg, R. Single-cell RNA-seq reveals dynamic, random monoallelic gene expression in mammalian cells. Science 343, 193196, doi:10.1126/science.1245316 (2014).

87 Schlitzer, A. et al. Identification of CDC1- and CDC2-committed DC progenitors reveals early lineage priming at the common DC progenitor stage in the bone marrow. Nat Immunol 16, 718-728, doi:10.1038/ni.3200 (2015).

$88 \mathrm{Gu}$, Z., Eils, R. \& Schlesner, M. Complex heatmaps reveal patterns and correlations in multidimensional genomic data. Bioinformatics 32, 2847-2849, doi:10.1093/bioinformatics/btw313 (2016).

$89 \mathrm{Li}, \mathrm{H}$. Aligning sequence reads, clone sequences and assembly contigs with BWA-MEM. (2013).

$90 \quad$ Picard toolkit. Broad Institute, GitHub repository, version 2.17.8, http://broadinstitute.github.io/picard/ (2018).

91 Zhang, Y. et al. Model-based analysis of ChIP-Seq (MACS). Genome Biol 9, R137, doi:10.1186/gb-2008-9-9-r137 (2008).

92 Quinlan, A. R. \& Hall, I. M. BEDTools: a flexible suite of utilities for comparing genomic features. Bioinformatics 26, 841-842, doi:10.1093/bioinformatics/btq033 (2010).

93 Grun, D., Kester, L. \& van Oudenaarden, A. Validation of noise models for single-cell transcriptomics. Nat Methods 11, 637-640, doi:10.1038/nmeth.2930 (2014).

94 Hastie, T., Tibshirani, R. \& Friedman, J. H. The elements of statistical learning : data mining, inference, and prediction. (2017).

95 Maaten, L. v. d. \& Hinton, G. Visualizing data using t-SNE. Journal of machine learning research 9, 2579-2605 (2008).

96 Mclnnes, L., Healy, J. \& Melville, J. UMAP: Uniform Manifold Approximation and Projection for Dimension Reduction. (2018).

97 Jacomy, M., Venturini, T., Heymann, S. \& Bastian, M. ForceAtlas2, a continuous graph layout algorithm for handy network visualization designed for the Gephi software. PLoS One 9, e98679, doi:10.1371/journal.pone.0098679 (2014).

98 Wolf, F. A., Angerer, P. \& Theis, F. J. SCANPY: large-scale single-cell gene expression data analysis. Genome Biol 19, 15, doi:10.1186/s13059-017-1382-0 (2018).

99 Lun, A. T., McCarthy, D. J. \& Marioni, J. C. A step-by-step workflow for low-level analysis of single-cell RNA-seq data with Bioconductor. F1000Res 5, 2122, doi:10.12688/f1000research.9501.2 (2016).

100 Jiang, H., Lei, R., Ding, S. W. \& Zhu, S. Skewer: a fast and accurate adapter trimmer for next-generation sequencing paired-end reads. BMC Bioinformatics 15, 182, doi:10.1186/1471-2105-15-182 (2014).

101 Dobin, A. et al. STAR: ultrafast universal RNA-seq aligner. Bioinformatics 29, 15-21, doi:10.1093/bioinformatics/bts635 (2013).

102 Picelli, S. et al. Full-length RNA-seq from single cells using Smart-seq2. Nat Protoc 9, 171-181, doi:10.1038/nprot.2014.006 (2014).

103 Chan, C. K. F. et al. Identification of the Human Skeletal Stem Cell. Cell 175, 43-56 e21, doi:10.1016/j.cell.2018.07.029 (2018).

104 Soneson, C., Love, M. I. \& Robinson, M. D. Differential analyses for RNA-seq: transcriptlevel estimates improve gene-level inferences. F1000Res 4, 1521, doi:10.12688/f1000research.7563.2 (2015). 
105 Satija, R., Farrell, J. A., Gennert, D., Schier, A. F. \& Regev, A. Spatial reconstruction of single-cell gene expression data. Nat Biotechnol 33, 495-502, doi:10.1038/nbt.3192 (2015).

106 Raouf, A. et al. Transcriptome analysis of the normal human mammary cell commitment and differentiation process. Cell Stem Cell 3, 109-118, doi:10.1016/j.stem.2008.05.018 (2008).

107 Pal, B. et al. Construction of developmental lineage relationships in the mouse mammary gland by single-cell RNA profiling. Nat Commun 8, 1627, doi:10.1038/s41467017-01560-x (2017).

108 Frazer, S. et al. Transcriptomic and anatomic parcellation of 5-HT3AR expressing cortical interneuron subtypes revealed by single-cell RNA sequencing. Nat Commun 8, 14219, doi:10.1038/ncomms14219 (2017).

109 Zhou, F. et al. Tracing haematopoietic stem cell formation at single-cell resolution. Nature 533, 487-492, doi:10.1038/nature17997 (2016).

110 Wu, B. et al. Reconstructing Lineage Hierarchies of Mouse Uterus Epithelial Development Using Single-Cell Analysis. Stem Cell Reports 9, 381-396, doi:10.1016/j.stemcr.2017.05.022 (2017).

111 Furlan, A. et al. Multipotent peripheral glial cells generate neuroendocrine cells of the adrenal medulla. Science 357, doi:10.1126/science.aal3753 (2017).

112 Chen, Y. J. et al. Single-cell RNA sequencing identifies distinct mouse medial ganglionic eminence cell types. Sci Rep 7, 45656, doi:10.1038/srep45656 (2017).

113 Joost, S. et al. Single-Cell Transcriptomics Reveals that Differentiation and Spatial Signatures Shape Epidermal and Hair Follicle Heterogeneity. Cell Syst 3, 221-237 e229, doi:10.1016/j.cels.2016.08.010 (2016).

114 Yang, L. et al. A single-cell transcriptomic analysis reveals precise pathways and regulatory mechanisms underlying hepatoblast differentiation. Hepatology 66, 13871401, doi:10.1002/hep.29353 (2017).

115 Li, L. et al. Single-Cell RNA-Seq Analysis Maps Development of Human Germline Cells and Gonadal Niche Interactions. Cell Stem Cell 20, 858-873 e854, doi:10.1016/j.stem.2017.03.007 (2017).

116 Hayashi, T. et al. Single-cell full-length total RNA sequencing uncovers dynamics of recursive splicing and enhancer RNAs. Nat Commun 9, 619, doi:10.1038/s41467-01802866-0 (2018).

117 Briggs, J. A. et al. Mouse embryonic stem cells can differentiate via multiple paths to the same state. Elife 6, doi:10.7554/eLife.26945 (2017).

118 Marques, S. et al. Oligodendrocyte heterogeneity in the mouse juvenile and adult central nervous system. Science 352, 1326-1329, doi:10.1126/science.aaf6463 (2016).

119 Qiu, W. L. et al. Deciphering Pancreatic Islet beta Cell and alpha Cell Maturation Pathways and Characteristic Features at the Single-Cell Level. Cell Metab 25, 11941205 e1194, doi:10.1016/j.cmet.2017.04.003 (2017).

120 Chan, C. K. et al. Identification and specification of the mouse skeletal stem cell. Cell 160, 285-298, doi:10.1016/j.cell.2014.12.002 (2015).

121 Wolock, S. L., Lopez, R. \& Klein, A. M. Scrublet: Computational Identification of Cell Doublets in Single-Cell Transcriptomic Data. Cell Syst 8, 281-291 e289, doi:10.1016/j.cels.2018.11.005 (2019).

122 Seita, J. et al. Gene Expression Commons: an open platform for absolute gene expression profiling. PLoS One 7, e40321, doi:10.1371/journal.pone.0040321 (2012). 


\section{Acknowledgments}

We thank A. Chaudhuri, A. Gentles, and A. Alizadeh for critical feedback on the manuscript. We are grateful to $S$. Bobo for assistance with patient specimen acquisition, R. Sinha and C.K.F. Chan for provision of data and resources, C.L. Liu for assistance with the website, P. Lovelace and S. Weber for their support and assistance with FACS, and S. Sim for assistance with processing and sequencing scRNA-seq libraries. This work was supported by grants from the National Cancer Institute (A.M.N., R00CA187192-03; M.F.C., 5R01CA100225-09; G.S.G., PHS Grant Number CA09302), the Stinehart-Reed foundation (A.M.N.), the Stanford Bio-X Interdisciplinary Initiatives Seed Grants Program (IIP) (A.M.N., M.F.C), the Virginia and D.K. Ludwig Fund for Cancer Research (A.M.N., M.F.C), the U.S. Department of Defense (M.F.C., W81XWH-11-1-0287 and W81XWH-13-1-0281; S.S.S., W81XWH-12-1-0020), a National Science Foundation Graduate Research Fellowship (DGE-1656518 to M.J.B.), Stanford Bio-X Bowes Graduate Student Fellowship (G.S.G.), and the Stanford Medical Science Training Program (G.S.G.).

\section{Author contributions}

G.S.G. and A.M.N. developed the concept for CytoTRACE, designed related experiments, and analyzed the data with assistance from S.S.S., D.J.W., and M.F.C. G.S.G. and A.M.N. wrote the manuscript with assistance from S.S.S. G.S.G. and A.M.N. performed the bioinformatics analyses with assistance from D.J.W., A.B., A.M., M.J.B., and F.L. A.M. and A.B. designed the website with input from G.S.G. and A.M.N. S.S.S. generated the human breast cancer singlecell RNA-sequencing data with assistance from A.H.K., R.W.H., S.C., M.Z., F.A.S., N.A.L., D.Q., and F.B.Y.S.S.S. performed the mouse experiments under the supervision of M.F.C. F.D. assisted with the collection of patient specimens. All authors commented on the manuscript at all stages.

\section{Data and materials availability}

Details of publicly available datasets are provided in Methods. Single-cell RNA-seq expression data generated in this study are hosted at https://cytotrace.stanford.edu with a GEO accession number pending. 

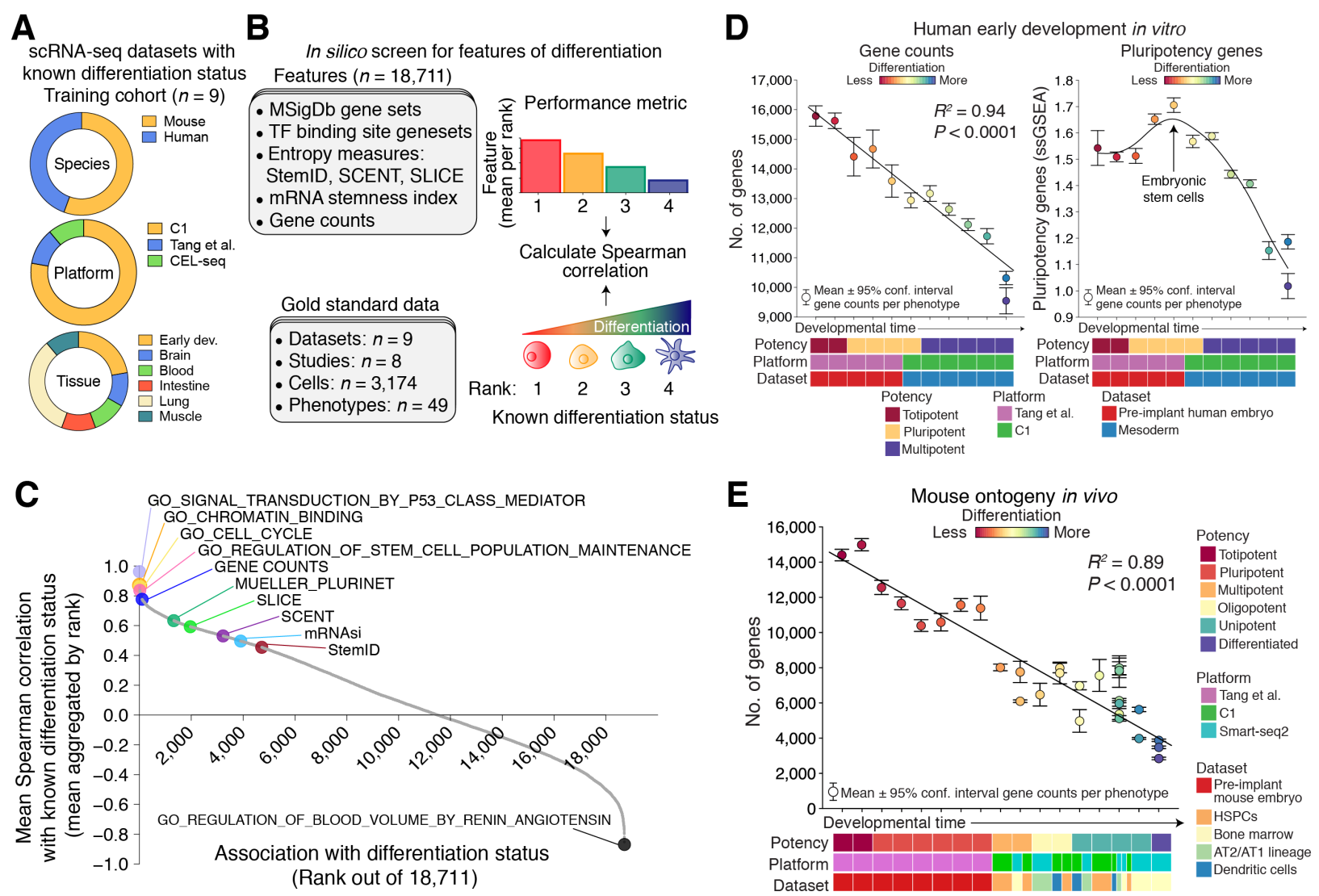

Figure 1 RNA-based determinants of developmental potential. (A-C) In silico screen for correlates of cellular differentiation status in scRNA-seq data. (A) Composition of the training cohort. (B) Left: Summary of evaluated features (top) and gold standard scRNA-seq datasets from the training cohort (bottom). In total, 17,810 gene sets from MSigDb, 896 transcription factor (TF) binding sites from ENCODE and ChEA, three measures of transcriptional entropy (StemID, SCENT, and SLICE), a machine learning model (mRNAsi), and gene counts (number of detectably expressed genes per cell) were assessed. Right: Depiction of the scoring scheme. Each phenotype was assigned a rank based on its known differentiation status (less differentiated = lower rank) and the values of each feature were mean-aggregated by rank for each dataset (higher value $=$ lower rank). Performance was calculated as the mean Spearman correlation between known and predicted ranks across all nine training datasets. (C) Performance of all features for predicting differentiation states in the training cohort, ordered by mean Spearman correlations. (D) The developmental ordering of 12 human cell phenotypes during early embryogenesis, shown as a function of single-cell gene counts (left) and the expression of pluripotency genes $\underline{21}$ (right). Points and error bars denote means and $95 \%$ confidence intervals, respectively. Phenotypes are ranked according to their known developmental potential relative to other cell types (phenotype labels are provided in Methods). The coefficient of determination $\left(R^{2}\right)$ is shown for gene counts (left) and a smooth spline is shown for pluripotency gene enrichment scores (right). ssGSEA, single sample gene set enrichment analysis. (E) Same as in D (left panel) but showing the ontological ordering of 30 mouse cell phenotypes across 17 developmental stages versus single-cell gene counts. For further details and information on datasets, see Methods. 
A

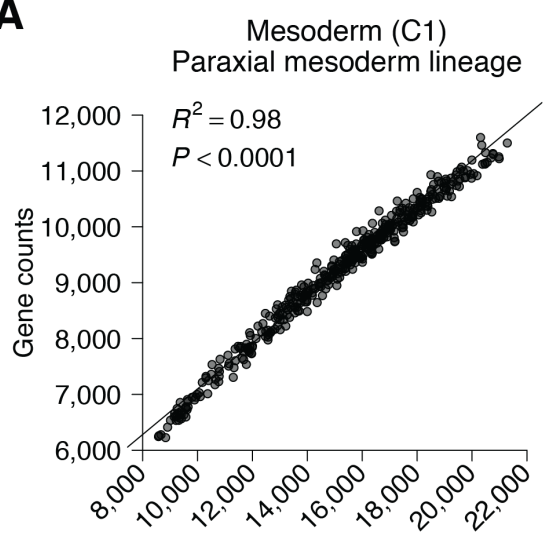

No. of unique splice isoforms
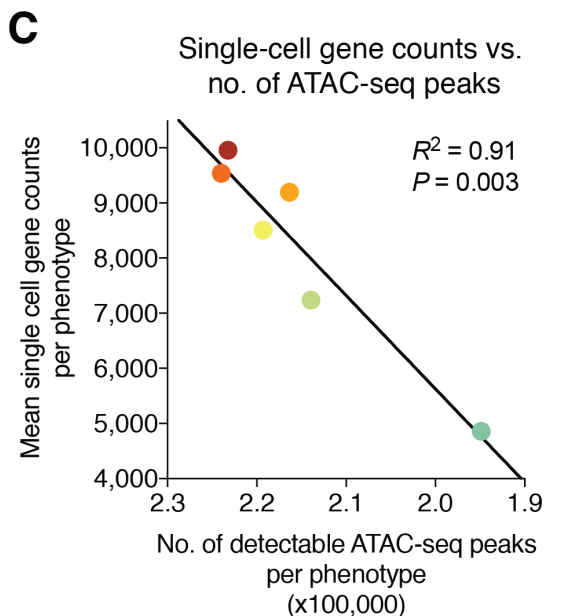

B
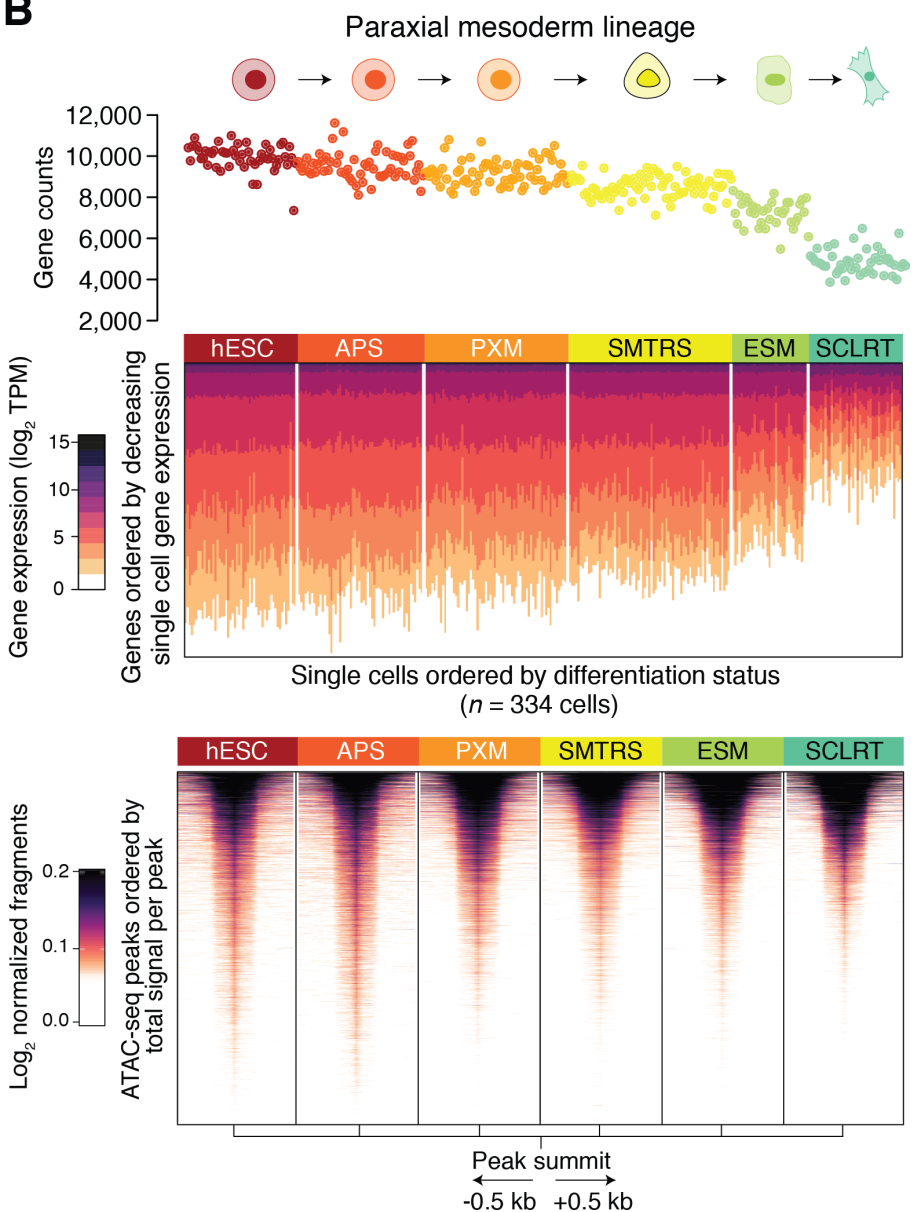

Figure 2 Association between gene counts, RNA diversity, and chromatin accessibility. (A-C) Analysis of the association between single-cell gene counts, transcriptional diversity, and chromatin accessibility in cells from an in vitro differentiation series of purified phenotypes from the human paraxial mesoderm lineage (Methods; also see Figure S7). (A) Scatterplot showing the association of gene counts with the number of unique protein-coding splice isoforms detected per cell. (B) Top: Scatterplot showing the association of gene counts ( $y$-axis) with paraxial mesoderm differentiation at the single-cell level ( $x$-axis). Each point represents a cell colored by known phenotype (below). Center: Heat map depicting each single-cell transcriptome in the above scatterplot, but ordered from top-to-bottom by decreasing gene expression $\left(\log _{2}\right.$ TPM). Cells are ordered left to right by increasing differentiation status. Bottom: Heat map showing chromatin accessibility profiles (bulk ATAC-seq) for the same cell phenotypes as above. Peaks are centered by their summit, defined as the base with maximum coverage, shown within a window of $1 \mathrm{~kb}( \pm 0.5 \mathrm{~kb})$, and ordered top to bottom within each phenotype by decreasing total signal per peak. (C) Scatterplot showing the concordance between the average number of single-cell gene counts per phenotype and the number of ATAC-seq peaks per phenotype. Points indicate cell types and are colored as in B. Linear relationships in A and C were evaluated by linear regression $\left(R^{2}\right)$ and corresponding $P$ values were determined by a $t$ test. hESC, human embryonic stem cell; APS, anterior primitive streak; PXM, paraxial mesoderm; SMTRS, somitomeres; ESM, early somites; SCLRT, sclerotome. 
A

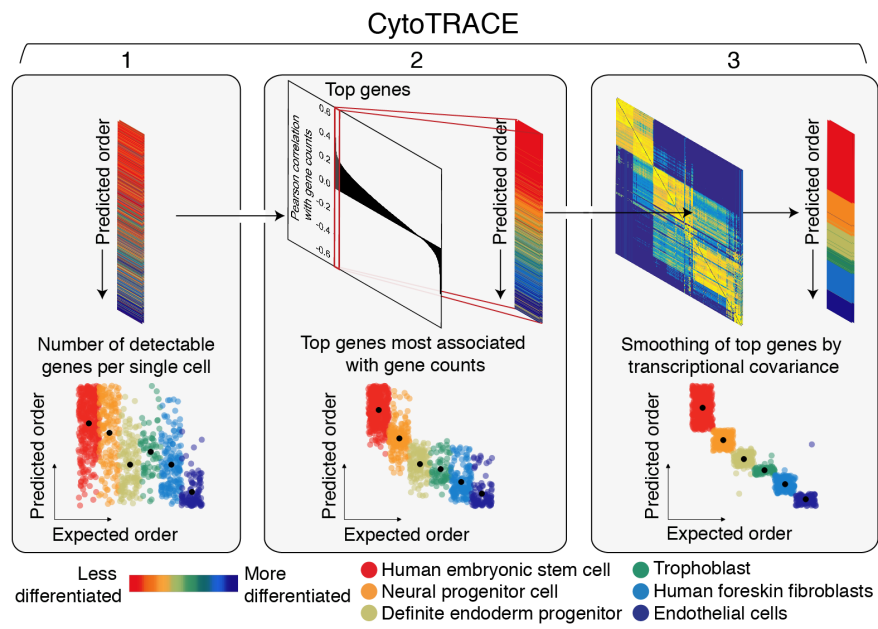

C

Performance of differentiation features against known ordering

- This study $(n=2)$

Stemness inference tools $(n=4)$

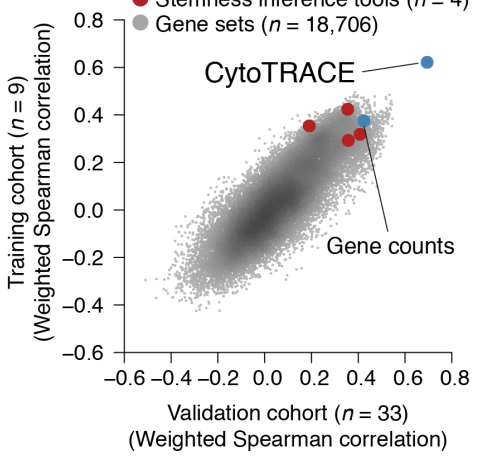

D

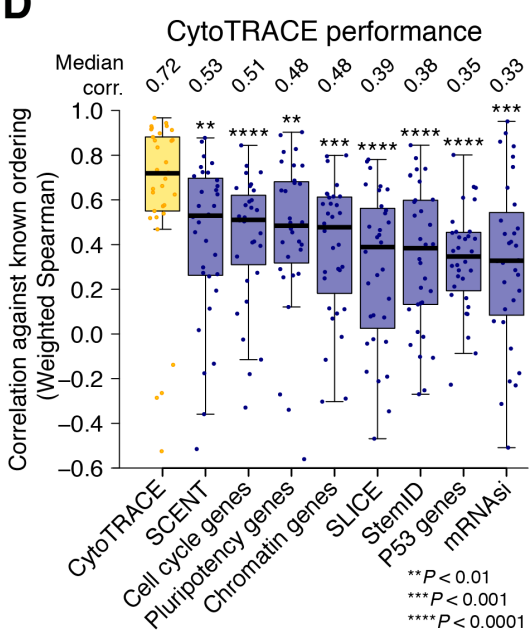

B

scRNA-seq datasets with known differentiation status Validation cohort $(n=33)$
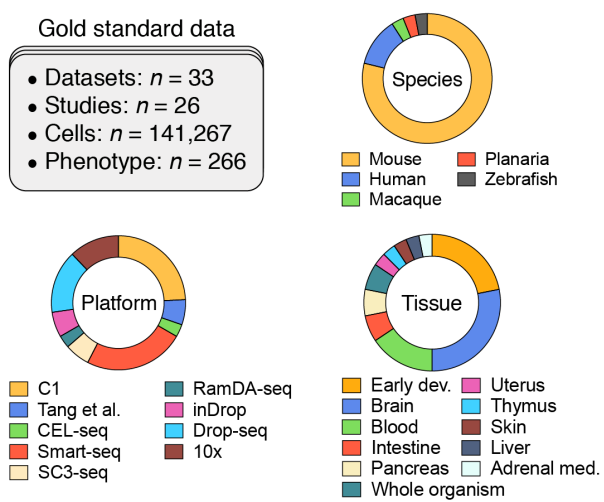

E

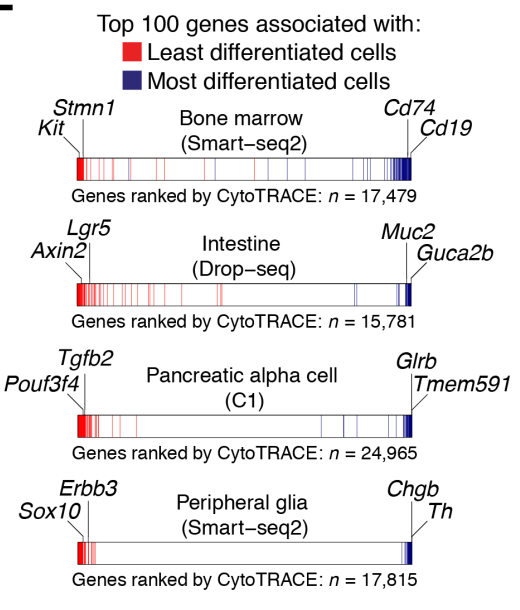

Figure 3 Development and validation of CytoTRACE. (A) Schematic overview of the CytoTRACE framework applied to the in vitro differentiation of hESCs into the gastrulation layers $^{39}$ (Methods). (B-D) Validation and benchmarking of CytoTRACE. (B) Composition of the validation cohort. In total, 33 scRNA-seq datasets with known differentiation states were analyzed. (C) Scatterplot showing predictive performance of 18,706 gene sets, four stemness inference methods $\frac{18-20,22}{}$, gene counts, and CytoTRACE, in the training cohort ( $y$-axis) and validation cohort ( $x$-axis). Results reflect the average single-cell performance per cohort. (D) Boxplots showing the single-cell level performance of CytoTRACE against the features and methods from Fig. 1B in the validation cohort ( $n=33$ datasets). Each point represents the Spearman correlation, weighted by number of cells per phenotype, between predicted and known differentiation states for a given dataset, calculated as described in Methods. Features and computational methods are ordered from left to right by median. Statistical significance was assessed by a one-sided paired Wilcoxon signed-rank test against CytoTRACE. (E) Developmental marker gene prioritization without prior knowledge of cellular phenotypes. Plots showing the enrichment of key stemness-associated (red) and differentiation-associated (blue) genes by CytoTRACE in bone marrow ( $n=4,897$ cells), intestine ( $n=7,216$ cells), pancreatic alpha cells; ( $n=338$ cells), and peripheral glia ( $n=369$ cells) (source data are described in Methods). Select markers of differentiation are indicated for each dataset. Also see Figure S12. 


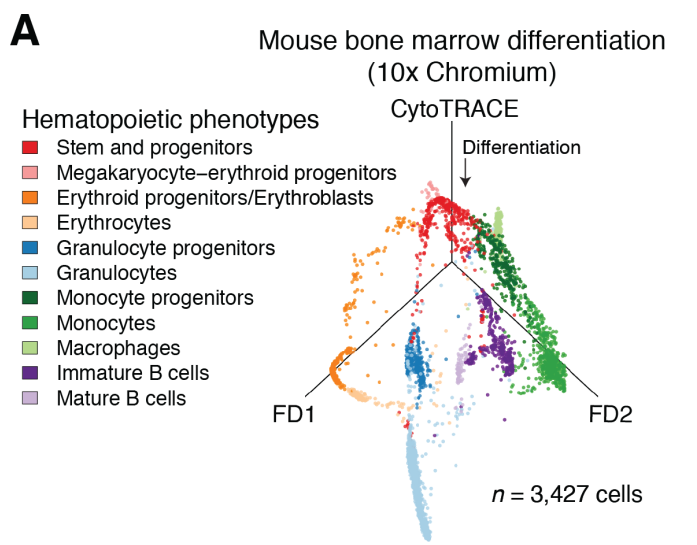

B

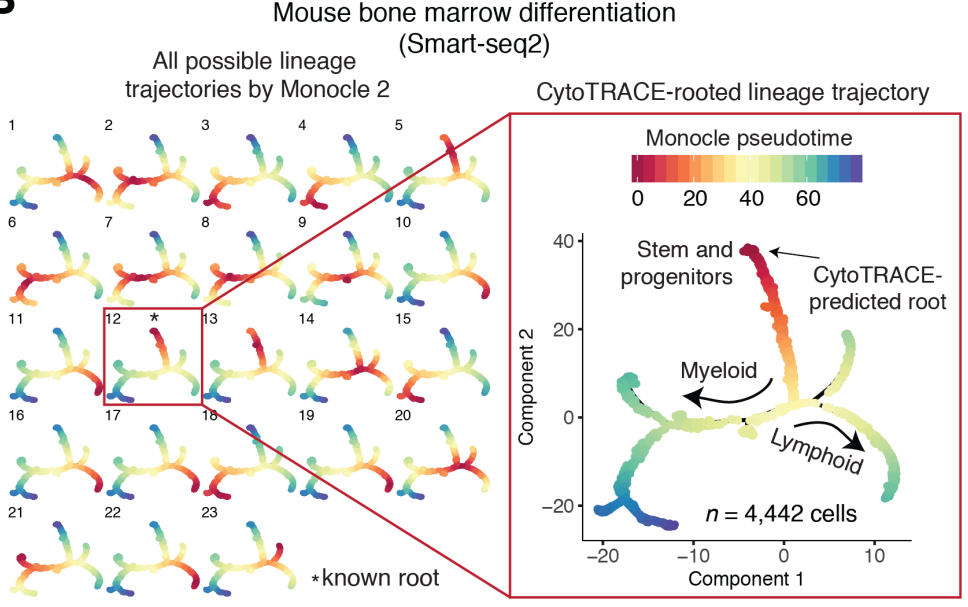

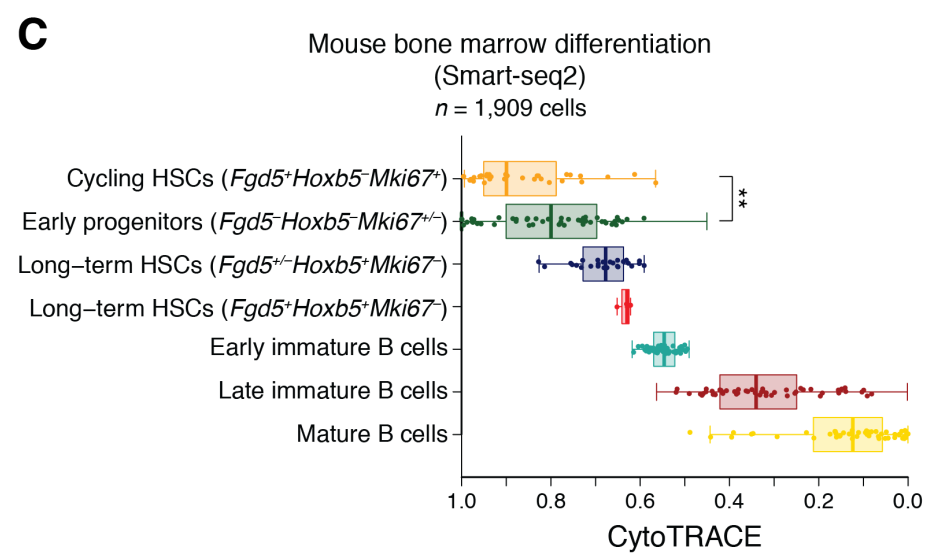

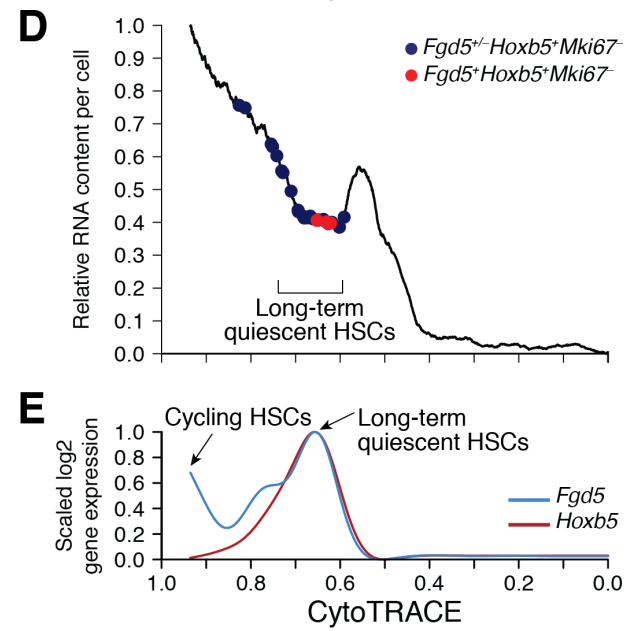

Figure 4 Characterization of developmental hierarchies and quiescent stem cells using CytoTRACE. (A-E) Application of CytoTRACE to dissect the mouse hematopoietic hierarchy by integrative analysis. (A) Mouse bone marrow scRNA-seq data visualized by CytoTRACE versus a force-directed layout (FD1 vs. FD2). (B) Combined application of CytoTRACE and Monocle 2 to delineate complex branches during mouse bone marrow differentiation without any prior information of the root. Multi-lineage tree inferred by Monocle 2 showing all 23 possible pseudotimes when the root is unknown (left) and automatic selection of the correct root by CytoTRACE (right). (C-E) Prioritization of quiescent and cycling hematopoietic stem cells (HSCs) in index-sorted scRNA-seq data of hematopoietic stem and progenitors $\left(\mathrm{KIT}^{+} \mathrm{SCA} 1^{+} \mathrm{LIN}^{-}\right.$ ) and developing B cells (TER119-B220 ${ }^{+}$. All plots are identically ordered, left to right, by CytoTRACE. (C) Boxplots showing CytoTRACE values for candidate cycling HSCs $(n=31)$ and long-term/quiescent HSCs $(n=30)$ versus early immature B cells $(n=285)$, late immature B cells $(n=863)$, and mature B cells $(n=700)$. HSC subgroups were defined based on expression of $F g d 5$, a reporter gene for $\mathrm{HSCs}^{67}$, Hoxb5, a reporter gene for long-term $\mathrm{HSCs}^{47}$, and Mki67, a marker of proliferation. Although boxplots represent all analyzed cells, for clarity, a maximum of 50 cells per phenotype are displayed as points. Statistical significance was assessed by an unpaired Wilcoxon signed-rank test. ${ }^{* *} P<0.01$. (D) Relative RNA content per cell, shown as a function of CytoTRACE ('Analysis of total RNA content and transcriptional diversity' in Methods) and displayed as the moving average of 200 cells. (E) Expression of Fgd5 and Hoxb5 ordered by CytoTRACE and displayed as a smoothing spline over the moving average of 200 cells. Data from monocytes and granulocytes (TER119-MAC1 ${ }^{+} \mathrm{GR} 1^{\text {high }}$ ) are consistent with the above results. Data in A and B-E are from 'Bone marrow (10x)' and 'Bone marrow (Smart-seq2)' datasets generated by Tabula Muris ${ }^{42}$, respectively (Methods). 
A

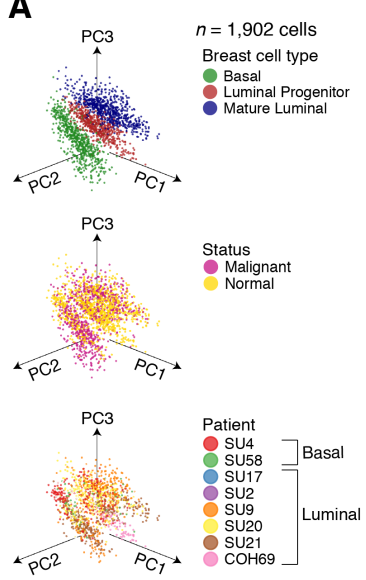

B
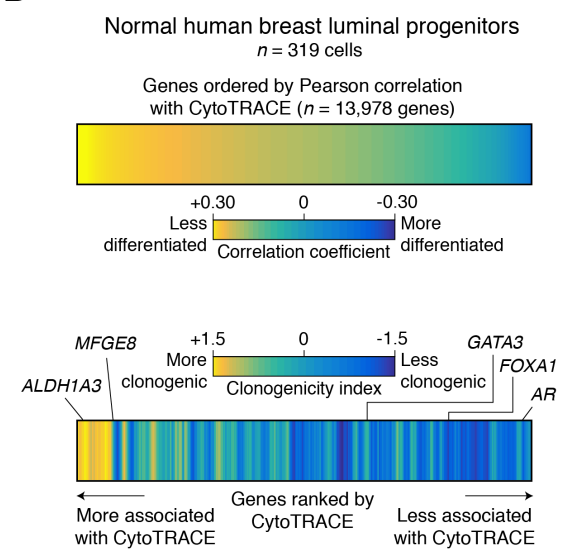

C
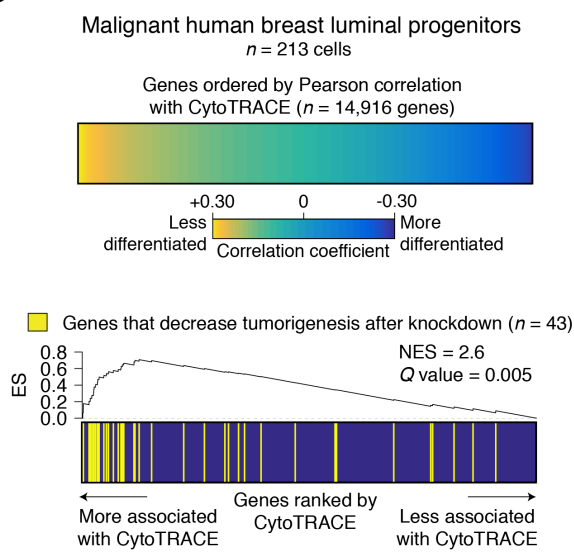

D

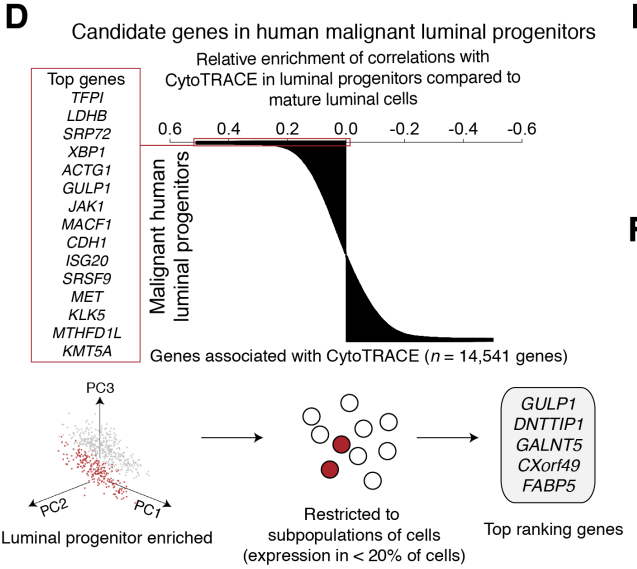

E

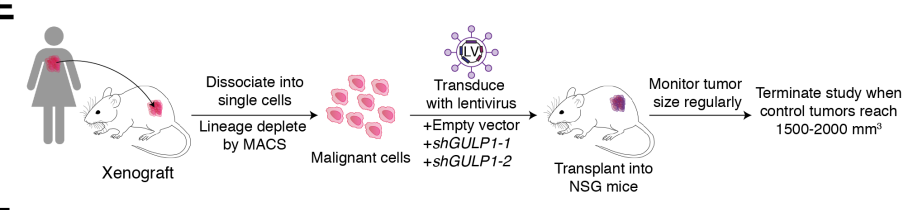

$\mathbf{F}$

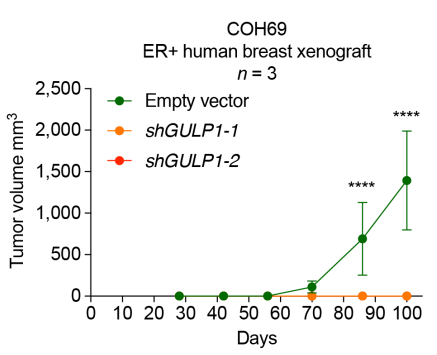

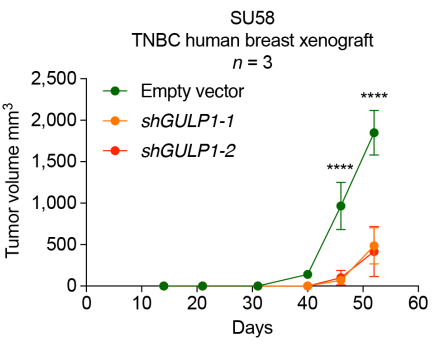

Figure 5 Identification of immature cell markers in normal and malignant human breast luminal progenitor cells using CytoTRACE. (A) Principal component analysis (PCA) plots showing scRNA-seq profiles of human basal cells ( $n=660$ cells), luminal progenitors $(n=532$ cells) and mature luminal cells ( $n=710$ cells) from 8 breast cancer patients ( 2 basal-like and 6 luminal-like), colored by epithelial subpopulations (top), tumor vs. paired adjacent normal tissues (center), and patient (bottom). (B) Prediction of differentiation-associated genes in normal luminal progenitors (LPs) profiled by scRNA-seq. Top: Heat map showing normal LP genes ordered by their Pearson correlation with CytoTRACE. Bottom: Heat map depicting the association of each gene in the above plot with a 'clonogenicity index', defined as the $\log _{2}$-fold change in expression between highly and lowly clonogenic LPs from normal human breast $\underline{\underline{54}}$ (Methods). The clonogenicity index is displayed as a moving average of 200 genes. Key genes associated with less (ALDH1A3, MFGE8) and more (GATA3, FOXA1, AR) differentiated normal LPs are indicated. (C) Prediction of differentiation-associated genes in malignant luminal progenitors profiled by scRNA-seq. Top: Same as panel B (top) but showing genes from malignant rather than normal LPs. Bottom: Pre-ranked gene set enrichment analysis $\underline{68}$ of 43 genes found to decrease human breast tumorigenesis in an RNAi dropout viability screen ${ }^{57}$ in relation to LP genes ranked by CytoTRACE (same order as above). NES, normalized enrichment score; ES, enrichment score. (D) Identification of candidate tumorigenic genes associated with less differentiated malignant human LPS. Top: Genes rank-ordered by the difference in their correlations with CytoTRACE in malignant LPs versus malignant mature luminal cells (MLs). The top 15 genes that are predicted to be specifically associated with less differentiated LPs are indicated (left). Bottom: Schema for the identification of genes that are ranked as above, but that are also more highly expressed in malignant LPs than MLs $\left(\log _{2}\right.$ fold 
change $>0$; Benjamini-Hochberg adjusted $P<0.05$, unpaired two-sided $t$-test) and that are expressed by a subpopulation of LPs ( $<20 \%$ of cells). The top 5 filtered genes are shown (right). (E) Schema for shRNA knockdown of GULP1 in a human breast cancer xenograft model. Lineage-depleted breast cancer epithelia cells from patient-derived xenografts were transduced with lentivirus containing either an empty vector control or shRNA targeting GULP1 and transplanted into immunodeficient NSG mice in triplicates. Tumors were monitored weekly until control tumors reached a size of $1500-2000 \mathrm{~mm}^{3}$. (F) Growth of human breast cancer xenografts from two patients, one with ER+ luminal-type cancer (left) and one with triplenegative breast cancer (right) after lentiviral transduction with empty vector or shRNA targeting GULP1. Mean tumor volume with $95 \%$ confidence interval is shown for 6 time points $(n=3$ mice). ${ }^{* * * *} P<0.0001$. 